\title{
The period-luminosity relation of Mira variable stars
}

\author{
Akiharu Nakagawa, ${ }^{1}$ Tomoharu Kurayama, ${ }^{2}$ Toshihiro Omodaka, ${ }^{1}$ \\ Tatsuya Kamezaki, ${ }^{1}$ Yoshiro Nishida, ${ }^{1}$ and Makoto Matsui ${ }^{1}$ \\ ${ }^{1}$ Faculty of Science, Kagoshima University, 1-21-35 Korimoto, Kagoshima, \\ Kagoshima 890-0065, Japan \\ email: nakagawa@astro.sci.kagoshima-u.ac.jp \\ ${ }^{2}$ Center for Fundamental Education, Teikyo University of Science, 2525 Yatsusawa, Uenohara, \\ Yamanashi 409-0193, Japan
}

\begin{abstract}
We use astrometry to measure the distances to Galactic Mira variable stars. Our purpose is to determine a precise period-luminosity relation (PLR). At present, we do not have a precise PLR for Galactic Mira stars because of the large uncertainties affecting their distance estimates. To reduce the uncertainties, we adopted the Very Long Baseline Interferometry method and measured annual parallaxes of Mira variable stars with VERA. In addition to our previous results, we obtained three new distances for Mira variable stars. Based on our observations, the typical uncertainty in a given distance measurement is reduced to below $10 \%$. At this conference, we present the current status of our project. To establish a precise Galactic PLR, we continue to observe more Mira variables. In addition, the apparent magnitudes of the target stars should be studied carefully.
\end{abstract}

Keywords. astrometry, stars: AGB and post-AGB, stars: variables: other 